\title{
HREM Observations of Continuously Changing Intermediate Structures between f.c.c. and b.c.c. at the Austenite-Martensite Interface
}

\author{
K. Ogawa, T. Kikuchi and S. Kajiwara \\ National Research Institute for Metals, 1-2-1 Sengen, Tsukuba 305, Japan
}

\begin{abstract}
Continuous lattice change from f.c.c. to b.c.c. as a function of subnanometric distance is observed near the austenite-martensite interface in an Fe-Ni-Mn alloy transformed isothermally at low temperature. From detailed analysis of such lattice changes, it is concluded that the lattice deformation proposed by Kurdjumov and Sachs is the most appropriate one. The Bogers-Burgers mechanism on which the Olson-Cohen nucleation model is based is found to be inadequate to explain the present observations.
\end{abstract}

\section{INTRODUCTION}

In the long history of study of martensitic transformation, a direct observation of the lattice change which generates the martensite structure from the parent structure is one of the most eagerly awaited achievements. It would be impossible to continuously observe such change with lapse of time, but, by using high resolution electron microscopy, we have recently succeeded in observation of the continuous change of the lattice with a function of subnanometric distance in the region near the parent-martensite interface. Due to the geometrical restriction, the observed lattice changes were those viewed along only one direction, but the results give us very important information on the lattice deformation in martensitic transformation. The actually observed sample is an Fe-Ni-Mn alloy which undergoes isothermal martensitic transformation from f.c.c. to b.c.c. at low temperatures. The essential part of the results was already published in the form of a letter [1], but, in this paper, more detailed description is given with a stereographic projection.

\section{EXPERIMENTAL METHOD}

An Fe-23.0Ni-3.8Mn (mass \%) alloy prepared by vacuum melting was used in the present experiment. Cold-rolled specimens $0.5 \mathrm{~mm}$ thick were heated for $30 \mathrm{~min}$ at $1070 \mathrm{~K}$ in evacuated capsules and quenched to room tempcrature. The quenched specimens were completely in austenitic state (f.c.c.) and those specimens were kept at $110 \mathrm{~K}$ for $7 \mathrm{~h}$ to be transformed isothermally into b.c.c. martensite. Thin foils for electron microscopy were prepared by electrolytic polishing at room temperature. High resolution electron micrographs were taken with a $200 \mathrm{kV}$ clectron microscope (JEOL 2000EX).

\section{EXPERIMENTAL RESULTS}

Figure 1 shows the structure images of the austenite $(\gamma$, f.c.c. $)$ and the martensite $(\alpha$, b.c.c. $)$ near at the $\gamma / \alpha$, interface which is located along an edge-on plane indicated by two arrows. This micrograph represents a part of the region near the $\gamma / \alpha^{\prime}$ interface of a martensite plate which is entirely confined within an austenite grain. The martensite plate is $1.6 \mu \mathrm{m}$ long and $0.13 \mu \mathrm{m}$ thick at the thickest point, reducing its thickness towards the growth edge. The high resolution electron micrograph in Fig. 1 was taken in such condition that all the diffraction spots shown in Fig. 2 (a) were allowed to pass through an objective aperturc. The zone axes of the diffraction pattern in Fig. 1 (a) are $[101]_{c}$ and $[111]_{b}$ and it is evident from this pattern that $(11 \overline{1})_{\mathrm{f}}$ is parallel to $(10 \overline{1})_{b}$, which means that the K-S orientation relationship holds in the martensitic transformation of this alloy as reported previously [2]. (Hereafter, subscripts, f and b, denote f.c.c. and b.c.c. lattices, respectively.) Figure 2 (b) shows fast Fourier transform pattern obtained from the area shown in Fig. 1. Under the present imaging conditions, the atom rows of $[101]_{\mathrm{f}}$ and $[111]_{\mathrm{b}}$ and the $\gamma / \alpha$ ' interface plane which is close to $(12 \overline{1})_{\mathrm{f}}$ are all parallel to the incident beam. The 
most densely packed planes, $(11 \overline{1})_{\mathrm{r}}$ and $(1 \overline{1} \overline{1})_{\mathrm{f}}$ in austenite, and $(10 \overline{1})_{\mathrm{b}}$ and $(1 \overline{1} 0)_{b}$ in martensite, are also parallel to the incident beam. The atom rows of $[101]_{\mathrm{r}}$ and $[111]_{\mathrm{b}}$ are clearly seen in Fig. 1 . The geometrical configurations of these atom rows, projected to a plane normal to the incident beam, is schematically shown in Figs. 3 (a) and (b). The observed spacings between these atom rows on the $\{111\}_{\mathrm{r}}$ and $\{110\}_{\mathrm{b}}$ plane are $0.22 \mathrm{~nm}$ and in good agreement with the calculated ones of $0.2192 \mathrm{~nm}\left(=\sqrt{6} / 4^{\bullet} \mathrm{a}_{\mathrm{f}}\right)$ and $0.2344 \mathrm{~nm}\left(2 / \sqrt{6} \cdot \mathrm{a}_{\mathrm{b}}\right)$ for f.c.c. phase and the martensite. Here $\mathrm{a}_{\mathrm{f}}$ and $a_{b}$ are lattice parameters of the austenite and the martensite of the present alloy and they were measured by $X$-ray diffraction as $\mathrm{a}_{\mathrm{f}}=0.3580 \mathrm{~nm}$ and $\mathrm{a}_{\mathrm{b}}=0.2870 \mathrm{~nm}$.

In order to more closely examine atomic arrangements near the interface, an inverse Fourier transform image of Fig. 1 was produced by using limited numbers of spots indicated by white circles in the Fourier transform pattern shown in Fig. 2(b), and an enlarged micrograph of the image is shown in Fig. 4. Two important features are noted in this figure. Firstly, all the lattice planes of two phases which are parallel to the incident beam are completely coherent at the interface. Sccondly, $(1 \overline{1} \overline{1})_{\mathrm{f}}$ plane is gradually curved into $(1 \overline{1} 0)_{\mathrm{b}}$ plane on crossing the interface. The latter fact suggests that, near the interface, there exist deformed lattices which are neither f.c.c. nor b.c.c.. For more direct visualization of those deformed lattices, an extremely enlarged micrograph of a part of Fig. 4 is shown in Fig. 5. We can notice in this micrograph that the configuration of the atom rows changes gradually from that of Fig. 3 (a) to that of Fig. 3 (b) when we trace from $P$ to $Q$ along $(11 \overline{1})_{\mathrm{f}}$ plane, or from $R$ to $S$ along $(1 \overline{1} \overline{1})_{\mathrm{f}}$ plane, passing through $O$. An intermediate shape between Figs. 3 (a) and (b) is clearly seen at $O$. To know the extent of the deformation in these lattices near the interface, the angle between $(1 \overline{1})_{\mathrm{f}}$ and $(1 \overline{1} \overline{1})_{\mathrm{f}}$ in austenite and the corresponding angle between $(10 \overline{1})_{b}$ and $(1 \overline{1} 0)_{\mathrm{f}}$ in martensite were measured along lattice planes indicated by arrows, a, b, c and d, and along lattice planes indicated by arrows, e, f, $g$ and $h$ in Fig. 4. The measured angles are shown in Figs. 6 (a) and (b), respectively. In Fig. 6 (a), the angles are plotted along $(1 \overline{1} \tilde{1})_{\mathrm{f}}$ plane which is normal to the interface plane, $(12 \tilde{1})_{5}$, while, in Fig. $6(\mathrm{~b})$, they are plotted along $(11 \overline{1})_{\mathrm{f}}$ plane which makes an angle of $19.5^{\circ}$ with the $(12 \overline{1})_{\mathrm{f}}$ interface. The abscissa in Fig. 6 (a) represents the distance along $[12 \overline{1}]_{\mathrm{f}}$ in austenite and along $[11 \overrightarrow{2}]_{\mathrm{b}}$ in martensite with the units of $\mathrm{a}_{\mathrm{f}} \sqrt{6 / 4}$ and $\mathrm{a}_{\mathrm{b}} 2 /$ $\sqrt{6}$, respectively, and, for Fig. 6 (b), it represents the distance along $[\overline{1} 21]_{\mathrm{f}}$ in austenite and $[\overline{1} 2 \overline{1}]_{\mathrm{b}}$ in martensite with the same units. (See Figs. 3 (a) and (b)). It is clearly seen in these figures that, on passing through the interface, the angle between $(11 \overline{1})_{\mathrm{f}}$ and $(1 \overline{1} \overline{1})_{\mathrm{f}}$ changes continuously from $70.5^{\circ}$ to $60^{\circ}$ which corresponds to the angle between $(10 \overline{1})_{b}$ and $(1 \overline{1} 0)_{b}$ planes. Therefore, we can say that there certainly exist transient lattices changing, continuously from f.c.c. to b.c.c.. From Fig. 6 (a), it is estimated that the region containing such transient lattices has a thickness of about $\mathrm{a} 3 \sqrt{6} / 4(=0.6576 \mathrm{~nm})$. It should be emphasized that this region constitutes the $\gamma / \alpha$ 'interface. It is seen from Figs. 3 (a) and (b) that these transient lattices can be brought about by simple shear of the f.c.c. lattice on (111) $)_{\mathrm{f}}$ plane in $[121]_{\mathrm{f}}$ direction as far as the configuration of the projected atom rows is concerned. The amount of the shear when the lattice is completely transformed from f.c.c. to b.c.c. is $1 / \sqrt{32}(=0.177)$, which is obtained from the geometry of the projected atom rows shown in Figs. 3 (a) and (b); that is, this shear is produced by the displacement of each $(11 \overline{1})_{\mathrm{f}}$ plane in $[\overline{1} 21]_{\mathrm{f}}$ direction by $\mathrm{a}_{\mathrm{f}} \sqrt{6} / 24$. The continuous angle change from $70.5^{\circ}$ to $60^{\circ}$ over a large distance in Fig. 6 (b) indicates more clearly the existence of various intermediate structures changing continuously from f.c.c. to b.c.c..

\section{DISCUSSION}

Although it was stated in the previous section that various stages of transient structures can be formed by simple shear on the $(11 \overline{1})_{\mathrm{f}}$ in the $[\overrightarrow{1} 21]_{\mathrm{f}}$ direction, this statement is only valid for the configuration of atom rows projected along the incident beam, namely, along $[101]_{\mathrm{f}}$ and $[111]_{b}$ directions. If we take into account atom size, such as hard spheres of atoms, which is known as a rigid sphere model, the shearing in $[121]_{\mathrm{f}}$ direction on the $(11 \overline{1})_{\mathrm{f}}$ plane is impossible to occur as shown in Fig. 7. Bearing in mind this point and based on the present observations of the atomic arrangements of the $\gamma / \alpha$ ' interface, we will discuss, in the following, which lattice deformation is plausible for the f.c.c. to b.c.c. transformation.

Three models which predict the K-S orientation relationship were proposed for the lattice deformation in the f.c.c. to b.c.c. martensitic transformation. They were by Kurdjumow and Sachs [3], by Frank [4] and by Bogers and Burgers [5]. Hereafter, these models will be called "K-S mechanism", "F-mechanism" and "B-B mechanism", respectively. To better understand the geometrical relations between those three mechanisms, a stereographic projection with the center pole of $[101]_{\mathrm{f}}$ and $[111]_{\mathrm{b}}$ is shown in Fig. 7 . The following description should be read referring to this figure. All of these mechanisms are basically comprised of two shears. When the K-S orientation 

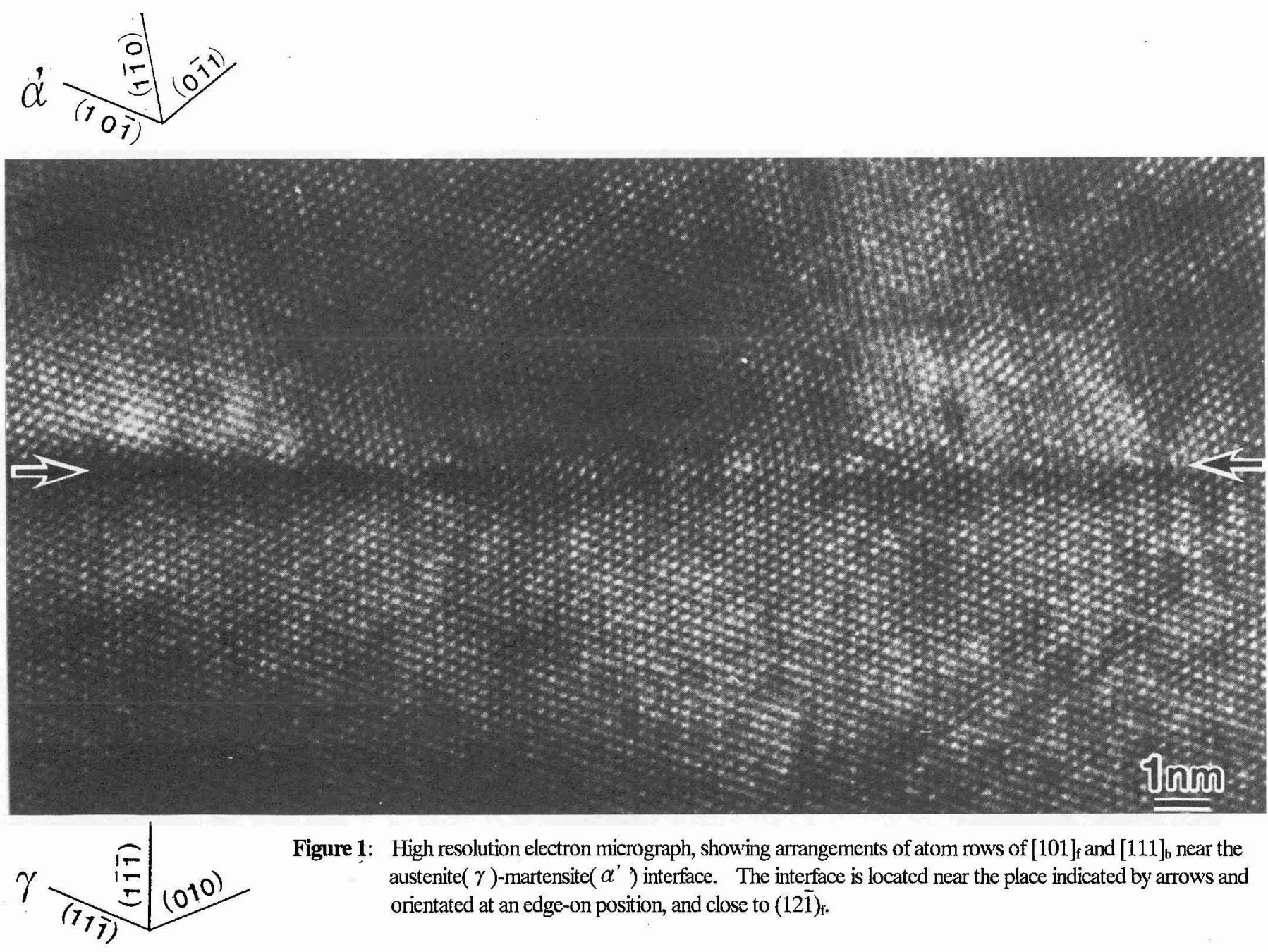

Figure 1: High resolution electron micrograph, showing arrangements of atom rows of [101 $]_{\mathrm{f}}$ and $[111]_{\mathrm{b}}$ near the austenite $(\gamma)$-martensite $\left(\alpha^{\prime}\right)$ interface. The interface is located near the place indicated by arrows and orientated at an edge-on position, and close to $(12 \overline{1})_{\text {. }}$. 

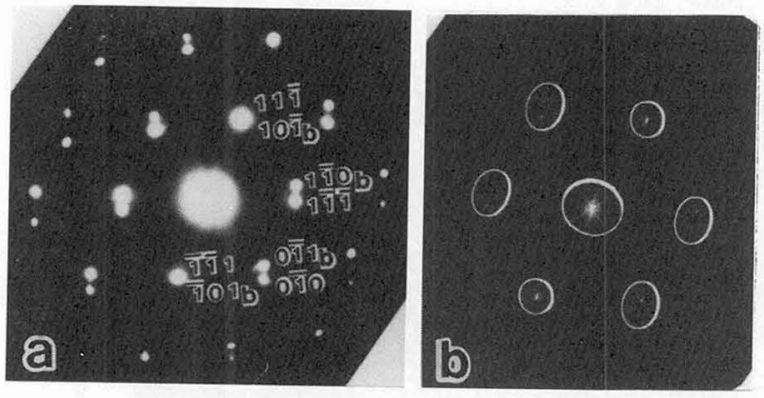

Figure 2: (a) Electron diffraction pattern taken from martensite plate and surrounding austenite. All the diffraction spots in this pattern were used for the formation of high resolution images.

(b) Fourier transform pattern produced from the images in Fig.1. (a)

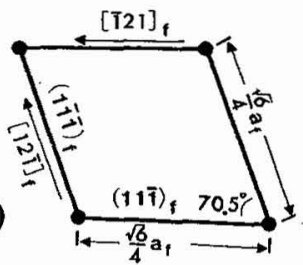

(b)

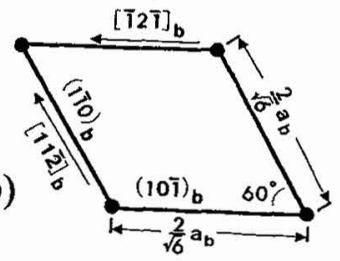

Figure 3: Schematic configurations of projected atom rows of $[101]_{\mathrm{f}}$ and [111]b.(a) f.c.c. lattice, (b) b.c.c. lattice.

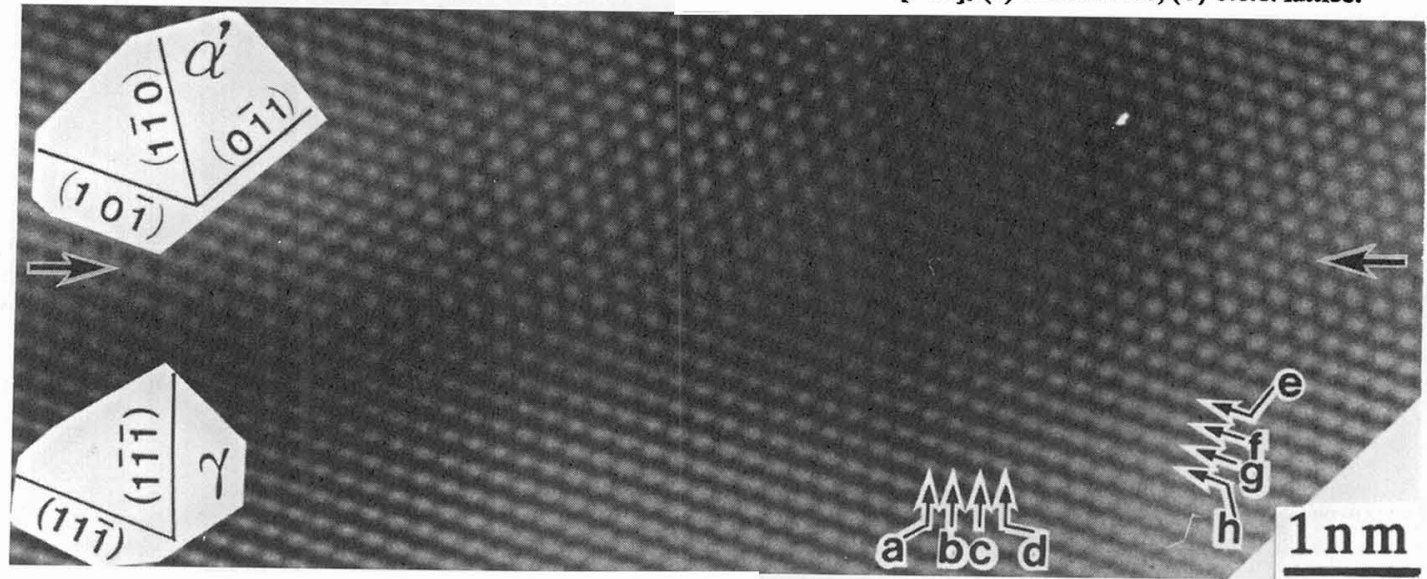

Figure 4: Inverse Fourier transform image of Fig. 1 produced by using spots encircled in Fig. 2 (b). Arrows indicate the position of the interface.

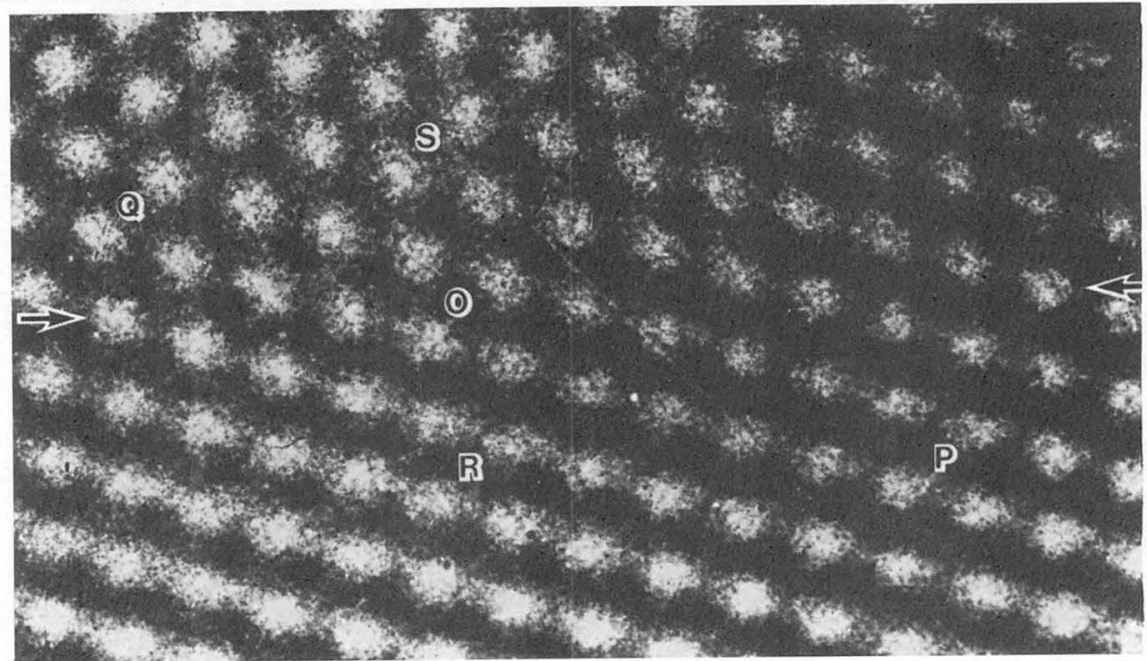

Figure 5: Extremely enlarged micrograph of Fig. 4, showing gradual change of the lattice. 

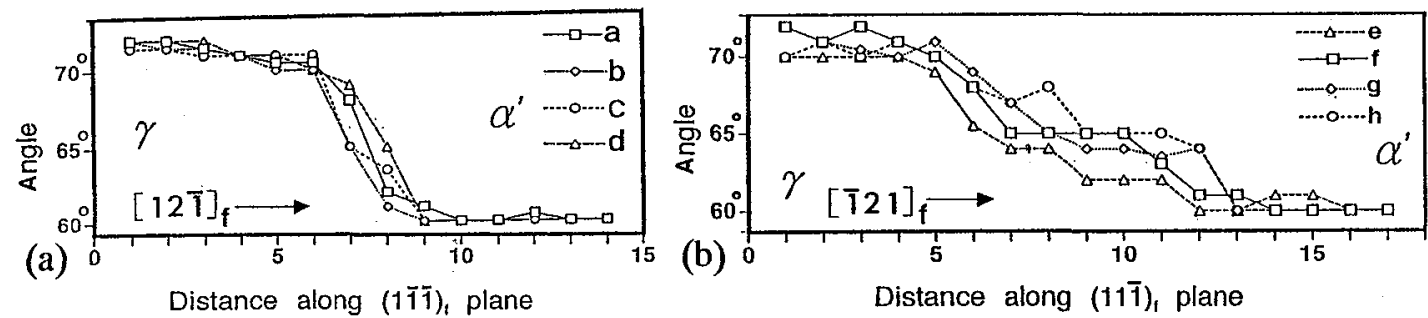

Figure 6: Change in angle between $(11 \overline{1})_{\mathrm{f}}$ and $(1 \overline{1} \overline{1})_{\mathrm{f}}$ when crossing the interface.

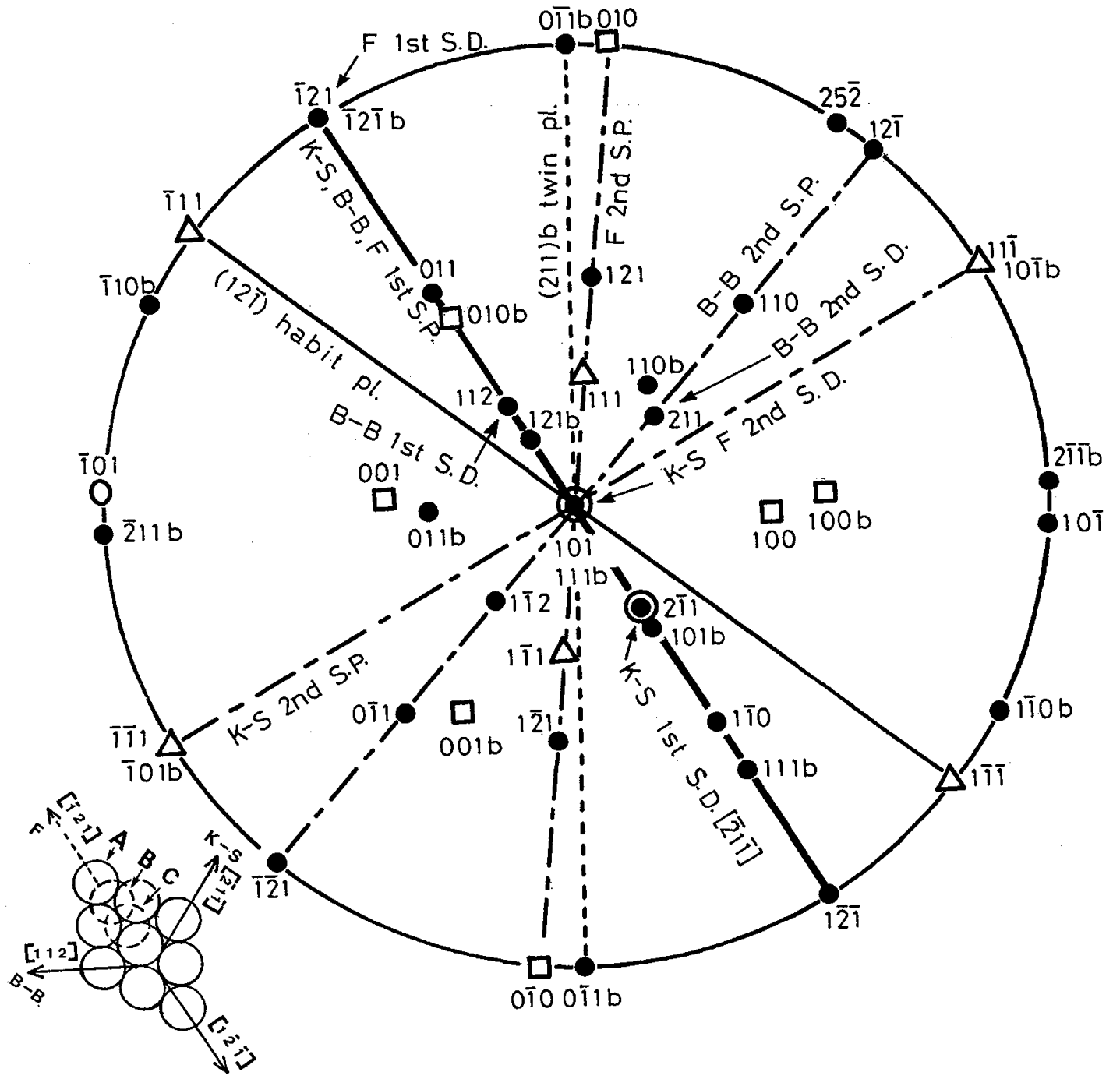

Figure7: Stereographic projection corresponding to Figs. 1 and 2. Indices without subscripts are for f.c.c. and those with subscript $b$ are for b.c.c. The center pole is taken parallel to 101 and $111_{b}$, namely, to the incident beam direction. K-S, F and B-B stand for Kurdjumov-Sachs, Frank and Bogers-Burgers mechanisms, respectively.S. P. and S.D. stand for shear plane and shear direction. Thick great circle shows the 1st common shear plane for K-S, F and $\mathrm{B}-\mathrm{B}$, which have $11 \overline{1}$ and $10 \overline{1}_{\mathrm{b}}$ poles. The atomic arrangement of $(11 \overline{1})_{\mathrm{f}}$ plane in f.c.c. structure is shown in the left lower comer. 
relationship is described as $(11 \overline{1})_{d} /(10 \overline{1})_{b},[101] / /[111]_{b}$, as adopted in the present paper, the first and second shcars in the K-S mechanism are $[\overline{2} 1 \overline{1}]_{\mathrm{f}}(11 \overline{1})_{\mathrm{f}}$ and $[101]_{\mathrm{f}}(1 \overline{2} \overline{1})_{\mathrm{f}}$, respectively, and those in F-mechanism are $[\overline{1} 21]_{(}(11 \overline{1})_{\mathrm{f}}$ and $[101]_{(}(20 \overline{2})_{\mathrm{f}}$ and those in the B-B mechanism are [112] $(11 \overline{1})_{\mathrm{f}}$ and $[211]_{(}(1 \overline{1} \overline{1})_{\mathrm{f}}$. The amounts of these shears in the K-S mechanism are 0.354 for the first shear and 0.199 for the second shear, which are produced by a shear displacement of $\mathrm{a}_{\mathrm{f}} / 12 \cdot[\overline{2} 1 \overline{1}]_{\mathrm{f}}$ on every $(11 \overline{1})_{\mathrm{f}}$ plane and a shear displacement $\mathrm{a}_{\mathrm{f}} / 12 \cdot[101]_{\mathrm{f}}$ on every third $(\overrightarrow{2} 42)_{\mathrm{f}}$ plane. The first and second shears in the F-mechanism are 0.177 and 0.333 , respectively, which are produced by a shear displacement of $a_{f} / 24 \cdot[\overline{1} 21]_{\mathrm{f}}$ on every $(11 \overline{1})_{\mathrm{f}}$ plane and a shear displacement of $\mathrm{a}_{\mathrm{f}} / 2 \bullet^{\bullet}[101]_{\mathrm{f}}$ on every $(20 \overline{2})_{\mathrm{f}}$ plane. The first shear and second shears in the B-B mechanism are 0.236 and 0.250 , respectively, which are produced by a shear displacement of $\mathrm{a}_{f} / 18 \cdot[112]_{\mathrm{f}}$ on every $(11 \overline{1})_{\mathrm{f}}$ plane and a shear displacement of $\mathrm{a}_{\mathrm{b}} / 8 \cdot[110]_{\mathrm{b}}$ on every $\left(1 \overline{1}(0)_{\mathrm{b}}\right.$ planc. The second shear in the B-B mechanism is equivalent to a shear displacement of $a_{f} / 16^{\bullet}[211]_{\mathrm{f}}$ on cvcry (11 $\left.1 \overline{1}\right)_{\mathrm{f}}$ planc. From a crystallographic analysis using the stereographic projection of Fig. 7, we can know that, in the K-S mechanism, the shear component of the first shear along [121] on (111) plane is 0.177 . This value is exactly the same as that of the observed amount of the apparent shear in $[\overline{1} 21]_{\mathrm{f}}$ on the $(11 \overline{1})_{\mathrm{f}}$ plane, which is deduced from the arrangement of atom rows projected to the plane normal to the incident beam. (The incident beam is parallel to $[101]_{\mathrm{f}}$ and $[111]_{\mathrm{b}}$ ) Moreover, in the K-S mechanism, the shear direction of the second shear is parallel to the incident beam, so that the atom displacement along this direction does not affect the image formation of the atomic structures in clectron microscope and, consequently, the positions of atom rows projected along the incident beam are expected to remain unchanged even if the austenitic crystal is subjected to the second shear. In other words, if the K-S mechanism is working, the high resolution electron microscope image of the atom rows along [101 $]_{\mathrm{f}}$ and $[111]_{\mathrm{b}}$ will remain unchanged even in the region near the $\gamma / \alpha$ ' interface where the transient structures from f.c.c. to b.c.c. appear. The undisturbed images of the atom rows near the interface actually seen in Figs. 4 and 5 strongly support the above supposition.

It is concluded from the following arguments that the other two mechanisms are not valid. Frank made a simple but serious mistake in constructing his model, namely, he thought that atoms can be moved in $[121]_{\mathrm{f}}$ direction on the $(11 \overline{1})_{\text {i }}$ plane. (See Fig. 3 and 7) However, as already mentioned before, shearing f.c.c. lattice directly into b.c.c. lattice along this direction is impossible. This is self-evident if we consider three dimensional configuration of f.c.c. structure as shown in Fig. 7. Therefore, we can exclude F-mechanism from the possible working mechanisms for the f.c.c. to b.c.c. lattice deformation. Finally we discuss the validity of the B-B mechanism. There are two reasons for dismissing this mechanism as inappropriate. Firstly, the shear component of the first shear in this mechanism along $[\overline{1} 21]_{\mathrm{f}}$ on $(11 \overline{1})_{\mathrm{f}}$ is 0.118 , which is much smaller than the observed one $(0.177)$. Secondly, if the transformation is assumed to occur according to this mechanism, high resolution images of the atom rows of [101 $]_{\mathrm{f}}$ and [111 $]_{b}$ near the $\gamma / \alpha$ 'interface would be disturbed because the second shear of this mechanism involves the atomic displacement which is not parallel to the incident beam but inclined by $30^{\circ}$. Thus we can conclude that the $\mathrm{K}-\mathrm{S}$ mechanism is the only plausible lattice deformation in the f.c.c. to b.c.c. martensitic transformation.

Finally we discuss briefly the nucleation model proposed by Olson and Cohen [6]. Their model is based on the B-B mechanism and two shears in the B-B mechanism were described by arrays of dislocations, mostly partial dislocations, at the interface of nucleating martensite. Besides the nucleation event itself, the kinetics of martensitic transformations in various Fe-based alloys, both athermal and isothermal, was analyzed in detail after this nucleation model (7). However, since the results of the present work have clearly shown that the B-B mechanism is not valid, the Olson-Cohen nucleation model have lost the validity of their basic assumption. Therefore, various phenomena concerning the nucleation of martensite and the kinetics of martensitic transformation must be reconsidered from the other bases and vicwpoints.

\section{References}

[1] S. Kajiwara, K. Ogawa and T. Kikuchi., Phil. Mag. Letters 74 (1996) 405.

[2] S. Kajiwara.., Phil. Mag. A 43 (1981) 1483.

[3] G. Kurdjumov and G. Sachs., Z. Phys., 64 (1930) 325.

[4] F. C. Frank., Acta Metall., 1 (1953) 15.

[5] A. J. Bogers and W.G. Burgers.,12 (1964) 255.

[6] G. Olson and M. Cohen.., Metall. Trans., 7A (1976) 1897, 1905, 1915.

[7] G. Olson and M. Cohen., Dislocations in Solids, Vol. 7, edited by F. R. N. Nabarro (Amsterdam: Noth Holland). 